\title{
Problems in Training: Swimming
}

Dr. H. N. Bleasdale, Hon. M.O., A.S.A.

Swimming is a sport of youth - there are nearly 500,000 competitive swimmers in Britain, and over 1,000 of them enter for the National Championships. They have a short championship life. A few remain in the sport and become coaches and officials, most of them leave and seek other interests.

The unit of swimming is the club. Led by a few dedicated officials and often short of funds, the club coaches the youngsters 2 or 3 evenings per week. Under these circumstances County or District representation may be gained. The dedicated ycungsters must swim once or twice daily in the active season. The public baths are crowded, and few pool managers are able to put a lane into the water during public hours. Many talented youngsters are unable to obtain the training necessary to achieve the highest standards of performance at the present time. The forthcoming facilities at Crystal Palace may be a fore-runner of a more realistic approach to this problem and perhaps may raise our standards to a new unforeseen peak. Whilst open air pools are too cold in Britain for training, a heated open air pool is ideal, the fresh air inhaled being better than the warm, humid indoor pool atmosphere. The temperature should be $74^{\circ} \mathrm{F}$ and the water must be treated in such a way that conjunctival hyperaemia does not occur after long swims. Other common ailments amongst swimmers due to mechanical washing of infection from nose and throat are sinusitis and otitis media.

The human body in water is not a good replica of the cigar shaped fish. We swim rather like the turtle - by means of paddles. The body should lie just below, and parallel to, the surface, avoiding, if possible, splash and surface turbulence which hinders speed. The shoulders should be strong. Atha has found a strong correlation between shoulder strength and speed. Hundreds of swimmers have been tested to discover their weaknesses physically and given remedial exercises to correct them. Flexibility is important, shoulders for backstroke and dolphin - hips, knees and ankles for freestyle and backstroke. If the swimmer is stiff, success may still be achieved by compensation in other ways by adjusting the stroke. 
Many swimmers use weight training and/or isometric training. These are good, but I am sure should be carried out daily or several times a week throughout the year. Wasting in muscle occurs soon, and swimming being only slightly weight bearing, does not produce adequate stimulus for hypertrophy to any great extent. Flexibility may be retained by exercises, but once lost, after midteens, is not easy to re-establish. Interval training is almost universally used to achieve cardio-respiratory efficiency and this can be continued on land by suitable circuits in the gymnasium. Apart from specialised skills involved in swimming movement, the swimmer may obtain his conditioning in varied ways, which add to the interest of the work and avoid the onset of staleness - these too promote fellowship which in turn produces team spirit.

Overseas, other problems enter the field. Long journeys, broken sleep, strange diet, or too much, varied climate - events held at times unsuitable to the individual, and some swimmers do badly in a morning - have all to be considered. Adequate time must be allowed for acclimatisation, and the use of a liquid or semi-solid meal prior to racing is worth consideration. Things in the world of swimming are good at present, but our eyes must look ever upwards.

\section{Problems in Training: Cycling}

Tommy Godwin, National Coach, B.C.F.

Injuries in cycling are not common and are usually due to crashes, being typically bruises, abrasions, with the occasional fracture. Some riders are apprehensive about anti-Tetanus injections and believe they affect performance.

With an increasing number of young people taking part in cycling we are worried about the possible harm that may come from overtraining, and also about the question of balanced development of the body in what is after all a highly specialised sport. These problems are underlined by the modern tendency to use more severe gear ratios.

There is some misunderstanding among cyclists and their trainers of the fundamentals of diet, particularly the role of vitamins, and we are as yet unable to give any authoritative guidance. 